\title{
Modelamiento Numérico Espacio-Temporal 1D de la Infiltración Basado en la Ecuación de Richards y Otras Simplificadas
}

\author{
Numerical Modeling Temporary-Space 1D of the Infiltration \\ Based on Richards's Equation and Other Simplified ones
}

\author{
'Edwin Pino Vargas, 2 Jesús Abel Mejía Marcacuzco
}

\begin{abstract}
RESUMEN:
La infiltración es uno de los procesos hidtrológicos que cobra unucha importancia en la ingenieria ambiental y de recursos hidricos, por décadas muchos investigadores han venido haciendo esfuerzos en modelar el proceso de infiltración, partiendo de la ecuación de Richards (1931). El comporlamiento de la infiltración puede ser tratado en forma tridimensional y tiempo en su forma más compleja, y dependiendo del uso que se requiera hasta en su forma unidimensional mas la componente temporal. En este irabajo se reduce la ecuación de Richards a su expresión unidimensional más su componente temporal y se resuelve bajo el método de diferencias finitas usando el esquema de Crank-Nicolson en un esquema implicito alterno exacto en segundo orden tanto en espacio como en tiempo. Dicho esquema fue codificado en MATLAB, y los resultados cumplen satisfactoriamente el objetivo de predecir el movimiento del agua en el subsuelo a partir de datos de propiedades fisicas de los suelos y condiciones impuestas tipo dirichlet de carga de agua sobre el suelo. Asimismo el modelo es muy versátil. puesto que permite establecer al usuario condiciones como profundidad total de simulación, espaciamiento entre mudos e intervalos de cálculo para la variable temporal. En el caso del modelo de Smith-Parlange (1978). fue resuelto usando el algoritmo de Newton Raphson. el mismo que también fue implementado en un código computacional en MATLAB, arrojando resultados satisfactorios similares a los del modelo anterior. Asimismo se elaboro un código computacional pararesolver el Modelo Green Ampt (1911), haciendo la comparación de los tres modelos mencionados
\end{abstract}

Palabras clave: ecuación de Richards, Sinith Parlage. Green Ampt, infiltración, modelamiento numérico ID

ABSTRACT:

Infiltration is one of the hydrological processes that receives great importance in the environmental engineering and water resources, per decades many investigators have been doing efforts modeling the process of infiltration, departing from the equation of Richards (1931). The behavior of the infiltration can be treated in three-dimensional and time in its more complex shapes, and depending on the required usage even in its one-dimensional form added the to temporal component. In this work we reduce Richards's equation to his unidimensional expression, added to his temporary component and it is solved according to the finite differences method using by Crank-Nicolson's scheme in an implicit alternate exact scheme, in the second order both in space and time. That scheme was codified in MATLAB, and the results fulfill satisfactorily the aim to predict the movement of water from the subsoil, from data of physical properties of the soils and imposed conditions dirichlet of water change on the soil. Likewise, the model is very versatile, hecause it allows the user to establish, conditions as total depth of simulation, spacing between knots and intervals of calculation for the temporary variable. In the case of the model of Smith-Parlange (1978), it was solved using the algorithm of Newton Raphson. it also was implemented in a computational code in MATLAB, giving satisfactory results similar to those of the previous model. Likewise, a computational code to resolve the Model Green Ampt (1911) was elaborated, doing the comparison between the three mentioned models.

Keywords: Richards's equation, Smith Parlage, Green Ampt, infiltration, numerical modeling $1 D$

\footnotetext{
' Master of Sciencie especialidad Ingenieria de Recurssos de Agua y Tierra, Ingeniero Civil, Ingeniero Agrícola

Facultad de Ingenieria Civil, Arquitectura y Geotecnia. Universidad Nacional Jorge Basadre Grohmann

2Doctor en Ingenieria Hidráulica(Brasil). Máster en Hidrologia (Bélgica), Magister en Ingenieria de Recursos Hidricos, Ingeniero Agricola.

Facultad de Ingenieria Agricola. Universidad Nacional Agraria La Molina
} 


\section{INTRODUCCIÓN}

El proceso de infiltración del agua en el suelo puede efectuarse tomando como base la ecuación de Richards (1931), que resulta de la combinación de la ecuación de continuidad con el campo de velocidades calculado conforme a la ley de Darcy.

La no linealidad de las propiedades del suelo como. conductividad hidráulica $\mathrm{K}$, difusión capilar D y capacidad especifica de agua en el suelo C, a evitado soluciones analiticas exactas, sin embargo actualmente el modelo conceptual de mayor aceptación es el modelo Richards.

La tasa de infiltración con respecto al tiempo, es gobernada por la ecuación de Richard, sujeta a una condición de humedad antecedente en el perfil del suelo, tasa de agua aplicada en la superficie del suelo y las condiciones de fondo del perfil del suelo. En general, el potencial inicial de agua en el suelo varia con la profundidad del suelo. Las condiciones iniciales $(t=0)$ pueden ser expresadas como un perfil de la carga potencial mátrico variando con respecto a la profundidad.

Las condiciones de borde de la superficie del suelo dependerán de la tasa de aplicación de agua. Para un evento de lluvia con intensidad menor o igual a la conductividad hidráulica en condiciones saturadas, toda la lluvia infiltrará al suelo y no se generará escurrimiento. Para lluvias de alta intensidad, toda la lluvia infiltrará en el suelo durante los primeros momentos hasta que la superficie del suelo se sature $(\Theta=\Theta s, \quad h \geq 0, \quad z=0)$ denominado tiempo de encharcamiento. Después de éste tiempo la infiltración será menor que la intensidad de la lluvia y se dará inicio al escurrimiento.

\section{METODOLOGÍA}

La solución de la ecuación de Richards hace indispensable representar las propiedades hidrodinámicas representado en el potencial de presión (h) y la conductividad hidráulica ( $\mathrm{K}$ ), como funciones del contenido volumétrico.

$\frac{\partial \theta}{\partial t}=\frac{\partial}{\partial x}\left(K \begin{array}{c}\partial h \\ \partial x\end{array}\right)+\frac{\partial}{\partial y}\left(K_{\partial y}^{\partial h}\right)+\frac{\partial}{\partial z}\left(K_{\partial z}^{\partial h}\right)+\frac{\partial K}{\partial z}$

Pavese et al. 1999, si bien el flujo en la zona no saturada es de carácter tridimensional no es difícil establecer que el flujo es principalmente vertical.

\section{Ecuaciones que Gobiernan el Proceso}

Durante las últimas décadas muchos investigadores se abocaron a la tarea de relacionar la humedad y la conductividad hidráulica no saturada con la tensión Gardner 1958, Brooks y Corey 1964, y Van Genuchten 1980, entre otros. En este modelo se utilizaron las fórmulas de Gardner para la determinación de los valores $\mathrm{K}(\mathrm{h})$ y $\theta(\mathrm{h})$..

$K(h)=K_{s} e^{\alpha h}$
Donde Ks ( $\mathrm{cm} / \mathrm{min})$ es la conductividad hidráulica del suelo saturado, $\alpha\left(\mathrm{cm}^{-1}\right)$ es un parámetro que caracteriza al tipo de suelo. La curva característica de humedad utilizada es una de tipo exponencial

$\theta(h)=\left(\theta_{s}-\theta_{r}\right) e^{\alpha / h}+\theta_{r}$

Donde $\theta$ s es el contenido de agua a saturación $\left(\mathrm{cm}^{3} \mathrm{~cm}^{-3}\right)$ y $\theta$ r es el contenido de agua a residual $\left(\mathrm{cm}^{5} \mathrm{~cm}^{-3}\right)$. La no linealidad representada por los parámetros $\mathrm{K}$ y $\mathrm{C}$ con la variable de estado $h$, se elimino parcialmente aplicando un cambio de la variable dependiente a través de la transformada integral de Kirchoff, introduciendo dicha transformada en la ecuación de Richards unidimensional se tiene:

$$
\frac{\partial u}{\partial t}+\begin{gathered}
1 \quad \partial K(h) \partial u \\
C(h) \quad \partial h \quad \partial z
\end{gathered}=\begin{aligned}
& K(h) \partial^{2} u \\
& C(h) \partial h^{2}
\end{aligned}
$$

Esta transformación fue previamente utilizada por Haverkamp and Vauclin (1981), y Kutilek et. al (1991), entre otros. Introduciendo las expresiones (2) y (3) en (4) y teniendo en cuenta que $\mathrm{C}(\mathrm{h})=\mathrm{dq}(\mathrm{h}) / \mathrm{dh}$, se obtiene:

$$
\frac{\partial u}{\partial t}+\frac{K_{s}}{\left.\theta_{s}-\theta_{r}\right)} \frac{\partial u}{\partial z}=\frac{K_{s}}{\left(\theta_{s}-\theta_{r}\right) \alpha} \frac{\partial^{2} u}{\partial h^{2}}
$$

Donde los coeficientes que preceden a las derivadas espaciales son constantes y función de los parámetros físicos del suelo. Además, de acuerdo a la transformación de Kirchoff y la relación de conductividad hidráulica adoptada y haciendo:

$$
C=\frac{K_{s}}{\left(\theta_{s}-\theta_{r}\right)}, \quad \gamma=\frac{K_{s}}{\left(\theta_{s}-\theta_{r}\right) \alpha}
$$

La ecuación de Richards en forma unidimensional con variación espacial temporal a resolver se reduce a:

$$
\frac{\partial u}{\partial t}+C_{\hat{\partial z}}^{\partial u}=\gamma \frac{\partial^{2} u}{\partial h^{2}}
$$

\section{Esquema Numérico de Solución}

La solución numérica de la ecuación (7) la podemos hacer usando el método de las diferencias finitas, por su facilidad de implementación. El avance temporal lo formulamos mediante el método de Crank-Nicolson. el que proporciona un esquema implícito alterno exacto en segundo orden tanto en espacio como en tiempo (Chapra 1998)

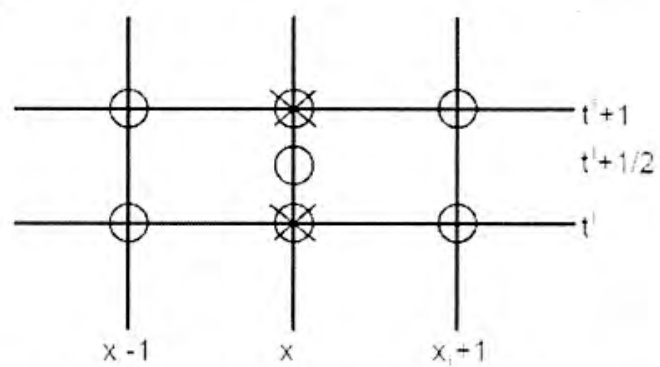

$X \quad$ Puntos de la malla involucrados en las diferencias en el tiempo

O Puntos de la malla involucrados en las diferencias en el espacio

Fig. 01 Esquema Computacional por el Método de Crank-Nicolson 
En el primer nudo interior para la aproximación temporal y espacial tenemos:

$$
\partial h=\begin{array}{cc}
h_{i}^{t+1}-h_{i}^{t} \\
\Delta t
\end{array} \quad C_{\partial z}^{\partial h}=C\left(\begin{array}{c}
h_{i+1}^{\prime}-h_{i-1}^{\prime} \\
2 \Delta z
\end{array}\right)
$$

La segunda derivada en el espacio corresponde al punto medio como promedio de las aproximaciones al inicio y final del incremento de tiempo:

$\gamma \frac{\partial^{2} h}{(\partial z)^{2}}=\gamma \frac{1}{2}\left(\begin{array}{c}h_{i+1}^{i}-2 h_{i}^{i}+h_{i-1}^{i}+h_{i+1}^{i+1}-2 h_{i}^{i+1}+h_{i-1}^{i+1} \\ (\Delta z)^{2}\end{array}\right)$

$-A h_{i-1}^{\prime+1}+2(1+A) h_{i}^{\prime+1}-A h_{i+1}^{\prime+1}=(A+B) h_{i-1}^{\prime}-2(A-1) h_{i}^{\prime}+(A-B) h_{i+1}^{\prime}$

$$
A=\begin{gathered}
y \Delta t \\
(\Delta=)^{2}
\end{gathered} \quad y=\begin{gathered}
C \Delta t \\
(\Delta-)^{2}
\end{gathered}
$$

Lacondición de frontera de $h_{o}^{t+1}=f_{o}\left(T^{t+1}\right)$

Puede ser prescrita para deducir el primer nudo interior. entonces este será:

$$
2(1+A) h h_{1}^{\prime+1}-A h_{2}^{\prime+1}=(A+B) f_{0}\left(T^{*}\right)-2(A-1) h_{1}^{\prime}+(A-B) h_{2}+A f_{0}\left(T^{*+1}\right)
$$

Para el último nudo interior no existe la condición de frontera:

$$
h_{m+1}^{\prime+1}=f_{m+1}\left(T^{\prime+1}\right)
$$

Por esta razón, para este nudo interior, se aplico las formulas de diferencias finitas divididas hacia atrás, de esta forma la primera derivada espacial fue aproximada en:

$$
C_{\partial z}^{\partial h}=C\left(\begin{array}{c}
h_{i}^{\prime}-h_{i-1}^{\prime} \\
\Delta z
\end{array}\right)
$$

Y la segunda derivada en el espacio fue determinada en el punto medio al promediar las aproximaciones al inicio y al final del incremento de tiempo.

$$
\gamma \underset{(\partial z)^{2}}{\partial^{2} h}=\gamma \frac{1}{2}\left(\begin{array}{c}
h_{i}^{\prime}-2 h_{i-2}^{\prime}+h_{i-1}^{\prime} \\
(\Delta z)^{2}
\end{array}+\begin{array}{c}
h_{i}^{i+1}-2 h_{i-2}^{i+1}+h_{i-1}^{i+1} \\
(\Delta-)^{2}
\end{array}\right)
$$

Sustituyendo las ecuaciones (8), (12) y (13) en la ecuación (7) se obtiene:

$$
\frac{h_{t}^{\prime+1}-h_{i}^{\prime}}{\Delta t}+C \frac{h_{i}^{\prime}-h_{i-1}^{\prime}}{\Delta t}=\gamma \frac{1}{2}\left(\frac{h_{i}^{\prime}-2 h_{t-2}^{\prime}+h_{t-1}^{\prime}}{(\Delta)^{2}}+\frac{h_{i}^{\prime+1}-2 h_{i-2}^{i+1}+h_{t-1}^{\prime+1}}{(\Delta)^{2}}\right) .
$$

Reagrupando términos tenemos:

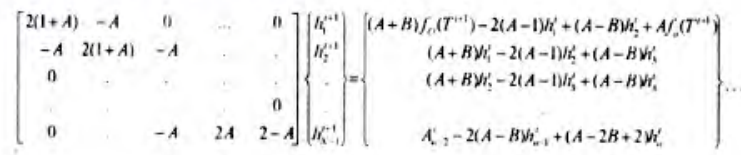

Las ecuaciones $(10)$ y $(15)$ son tridiagonales y por to tanto se pueden resolver de manera eficiente mediante el sistema de ecuaciones. Para la solución del sistema de ecuaciones planteado se elaboro un código computacional en MATLAB.

El Modelo Smith Parlange (1978), desatrollado por Woolhiser(1989), es la siguiente:

$f=K_{s} \frac{e^{F / B}}{e^{F / B}-1}$

Donde:

F: Infiltración acumulada [L], f: Velocidad de infïltración $[\mathrm{L} / \mathrm{T}]$. Ks: Conductividad hidráulica a saturación natural $[\mathrm{L} / \mathrm{T}], \mathrm{B}=\mathrm{G}(\theta \mathrm{s}-\theta 1) \circ \mathrm{B}=\mathrm{G} \varphi(\mathrm{S}$ max - S1). 0 s: Contenido de humedad a saturación natural ( $\mathrm{L} / \mathrm{L}$ ) , $\theta \mathrm{I}$ : Contenido de humedad inicial $\left((\mathrm{L} / \mathrm{L}), \varphi\right.$ : Porosidad del suelo $\left[\mathrm{L}^{\mathrm{t}} / \mathrm{L}\right]$. S: Saturación relativa. Smáx: Saturación relativa máxima. S1: Saturación relativa inicial y $\mathrm{G}$ es definida como:

$G=\frac{1}{K_{s}} \int_{-\infty}^{0} K(h) d h$

En la cual:

h: potencial de presión [L]. K(h): Conductividad hidráulica [L/T]. G: Potencial de escurrimiento o potencial de presión debido a capilaridad [L]. En el cálculo de la infiltración se debe elegir correctamente el primer estimador de $\mathrm{F}$ para que haya convergencia al utilizar el Newton-Raphson: un buen estimador es: $\mathrm{F}=\mathrm{K}$. t. y la aproximación de $\mathrm{F}$ se da según:

$$
F_{k+1}^{j}=F_{k}^{\prime}+\delta F^{J}
$$

Donde 'j' denota el paso de tiempo y ' $k$ ' la iteración respectivay

$\delta F^{J}=-r \partial r \partial F \quad \partial F=\begin{gathered}1-1 e^{i / n} \\ K\end{gathered}$

CURVA DE CONTENIDO DE AGUA PARA DIFERENTES TIEMPOS-RICHARDS

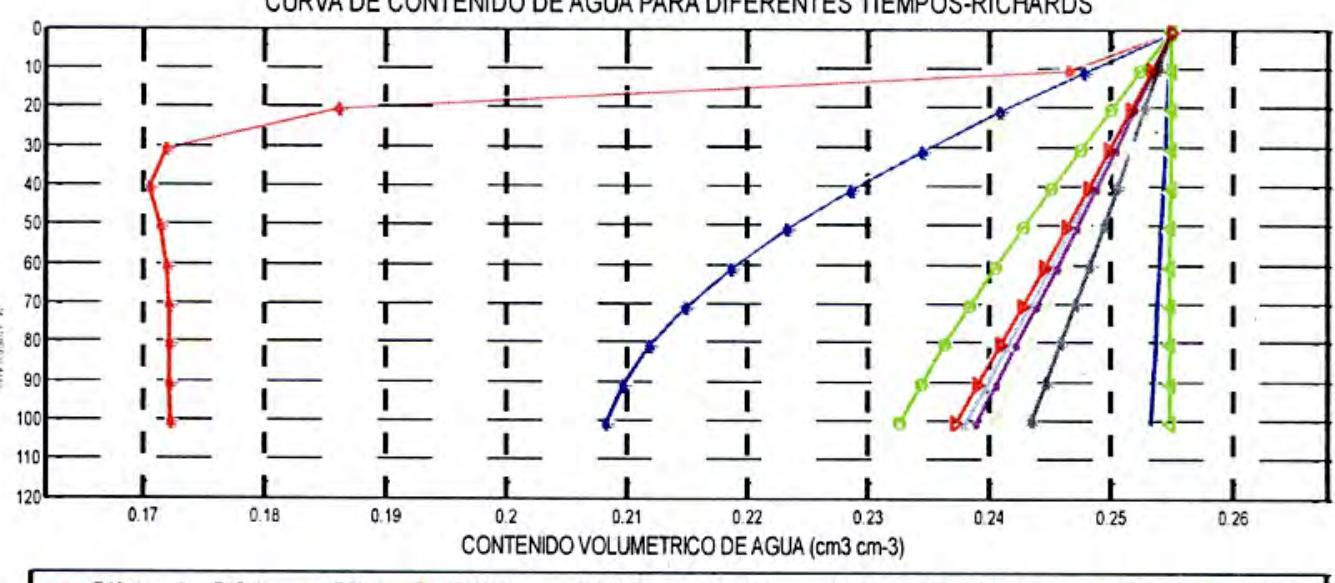

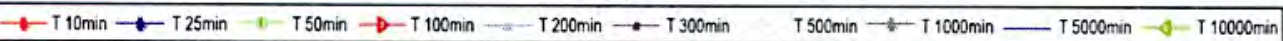

Fig. 02 Curva de contenido de agua para diferentes tiempos tomando como referencia la superficie del suelo. 


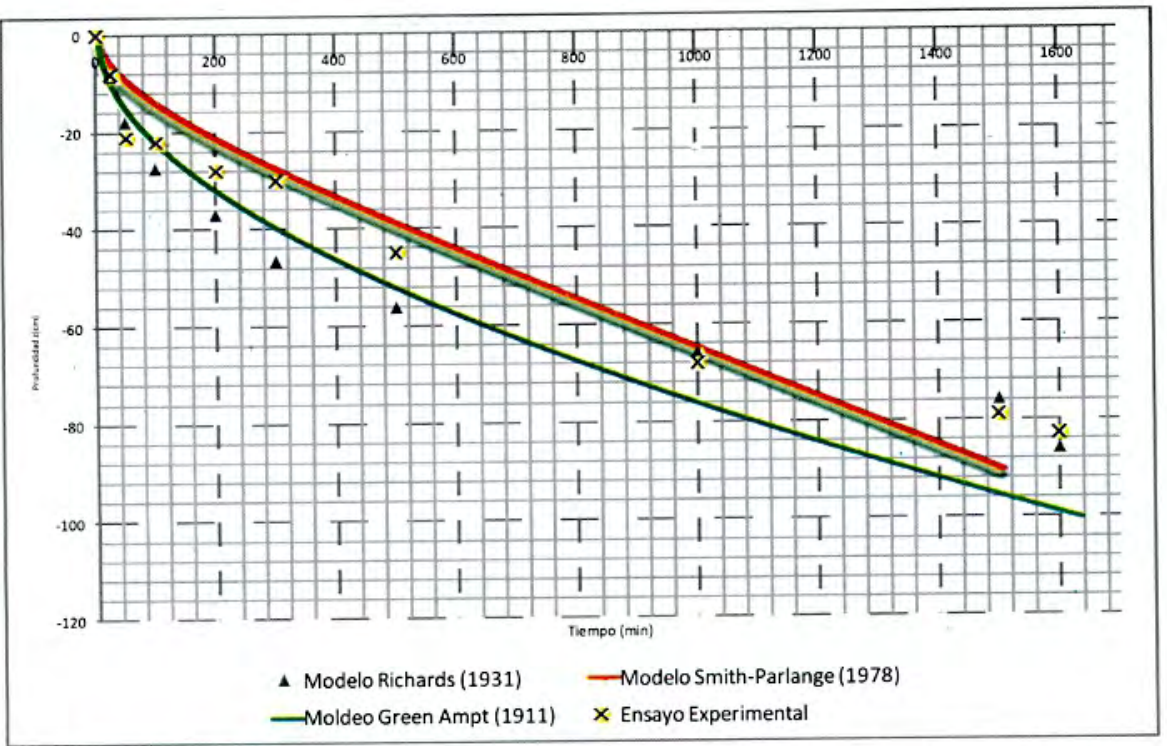

Fig. 03 Curva de desplazamiento del frente mojado para los tres modelos ensayados y datos experimentales tomados de Dahualde G. (2005)

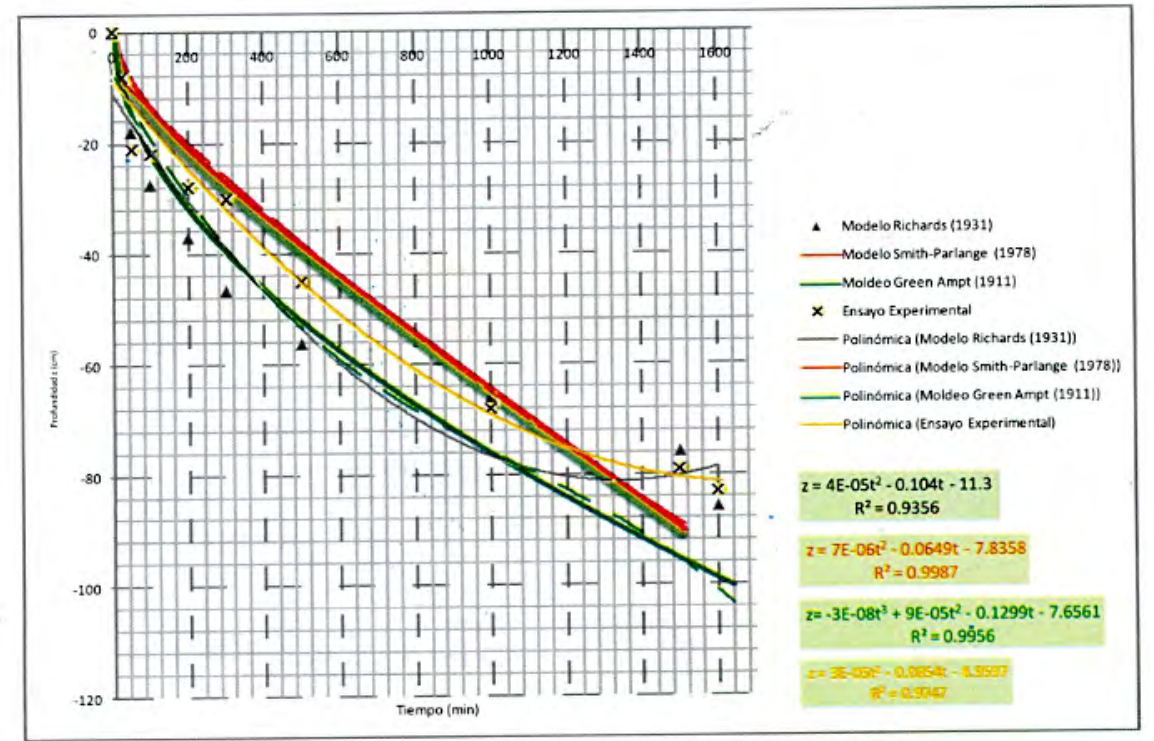

Fig. 04 Frentes de avance y análisis de regresión

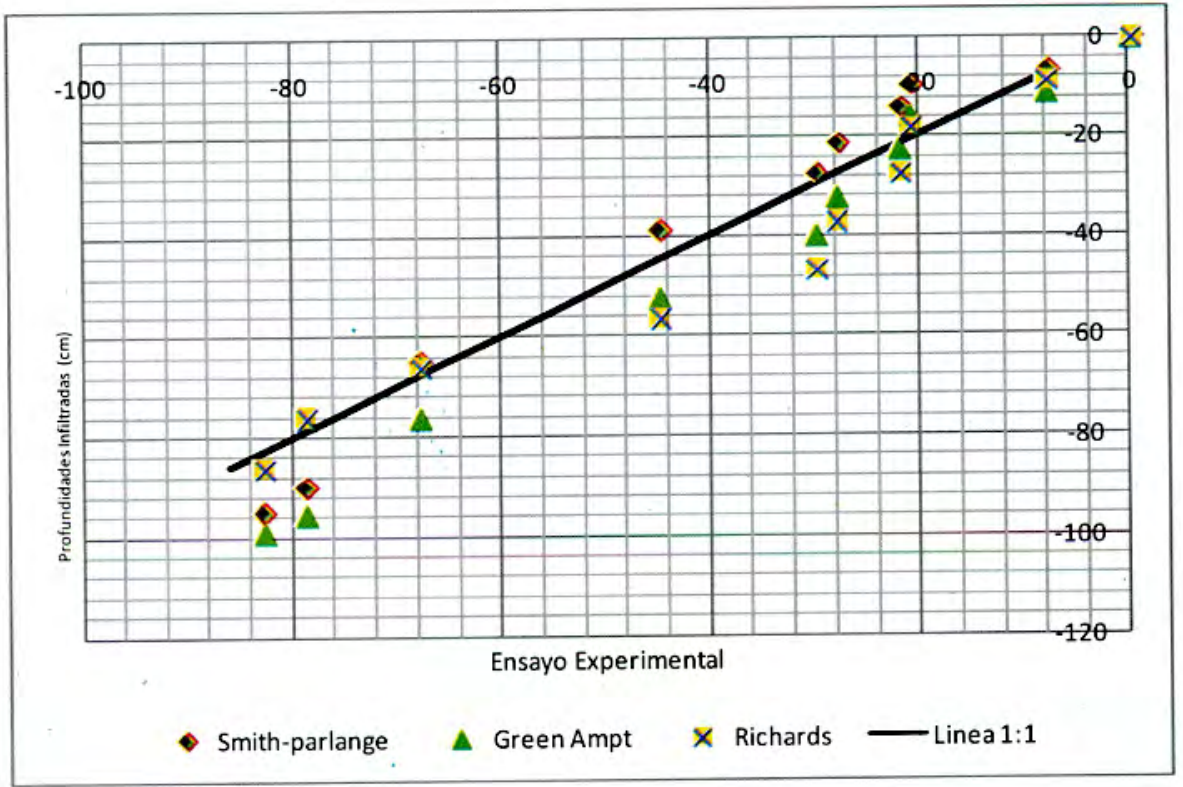

Fig. 05 Comparación gráfica de los resultados obtenidos 
Se conseguirá la solución cuando: $|\mathrm{r}|<=\mathrm{r}_{\max }$.

El Modelo Green Ampt (1911), fue el primer modelo físicamente basado en ecuaciones que describen la infiltración de agua en el suelo. Este modelo entrega la infiltración acumulativa y la velocidad de infiltración como funciones implicitas del tiempo.

Las ecuaciones tienen que ser resueltas de una manera iterativa para obtener estas cantidades. El modelo explícito Green-Ampt desarrollado por Salvucci y Entekhabi (1994), facilitó una estimación sincera y exacta de la infiltración para cualquier tiempo dado. Las formulaciones matemáticas para este modelo son las siguientes:

$\int_{d t}^{d F}=\int K \cdot\left(\begin{array}{c}\psi / \Delta \theta+F \\ F\end{array}\right)=F(t)-\psi / \Delta \theta \ln \left(1+\frac{F(t)}{y / \Delta \theta}\right)=K^{\prime \prime}$

La ecuación (20), es conocida como la ecuación de Green Ampt para la infiltración acumulada. Como se trata de un caso en el que se tiene encharcamiento en superficie, la variable $\psi$ debe ser reemplazada por ( $\psi$ $\mathrm{h}_{\mathrm{s}}$ ). Donde $\mathrm{K}$ es la conductividad, $\psi$ carga de succión de suelo en el frente de mojado, $\Delta \theta$ cambio en el contenido de humedad en el frente de avance.

\section{RESULTADOSY DISCUSIÓN}

Usando los modelos elaborados y el respectivo código computacional desarrollado en MATLAB (abreviatura de MATrix LABoratory, "laboratorio de matrices") que es un software matemático que ofrece un entorno de desarrollo integrado (IDE) con un lenguaje de programación propio (lenguaje $\mathrm{M}$ ), se procedió a efectuar procesos de simulación, para lo cual se tomo datos referenciales de un suelo de características Franco-Limoso, para lo cual se tiene los siguientes datos de entrada: $t=10,25,50,100,200,300,500,1000,5000$ y $10000 \mathrm{~min}$; Tiempo de Simulación. $z=100$; Profundidad de la columna de suelo a ser simulada $(\mathrm{cm})$. $\mathrm{Ks}=0.011$; Conductividad Hidráulica Saturada $(\mathrm{cm} / \mathrm{min}) . \theta \mathrm{s}=0.255$; Contenido de agua a saturación $\left(\mathrm{cm}^{3} / \mathrm{cm}^{3}\right) . \theta \mathrm{r}=0.045$; Contenido de agua a residual $\left(\mathrm{cm}^{3} / \mathrm{cm}^{3}\right) . \alpha=0.002 \mathrm{I}$; Constante alfa $\left(\mathrm{cm}^{-1}\right), \Delta \mathrm{t}=10$; Intervalo de tiempo para calculo ( $\mathrm{min}$ ). $\Delta z=10$; Discretización espacial $(\mathrm{cm})$. h $0=0$; Condición de borde, que puede ser sustituida por cualquier valor de carga hidráulica sobre la superficie del suelo, presión de succión $(\mathrm{cm}) . \mathrm{hl}=-1000$; Condición inicial, presión de succión $(\mathrm{cm})$.

Para observar los resultados obtenidos, visualizamos un gráfico que relaciona Profundidad $\mathrm{z}(\mathrm{cm})$ con el contenido de humedad volumétrico $\left(\mathrm{cm}^{3} \mathrm{~cm}^{-3}\right)$.

Los resultados obtenidos de la simulación muestran el contenido volumétrico de agua para condiciones medias de un suelo franco limoso, en función de la profundidad a diferentes tiempos: 10, 25, 50, 100, 200,300,500, 1000,5000 y $10000 \mathrm{~min}$. De lo cual podemos observar que los valores mínimos del contenido de agua inicialmente a lo largo del perfil del suelo tienden al valor del contenido de humedad residual que caracteristico en este tipo de suelo, luego van aumentando en el tiempo en forma descendente con respecto a la profundidad.

Asimismo el contenido de agua en el tiempo tiende al valor de este a saturación, por tanto mientras aumente el tiempo transcurrido el valor del contenido de agua tiende al valor a saturación, entendiéndose que el suelo estará saturado en cualquier punto a lo largo del perfil del suelo. Si observamos la figura 02 a $60 \mathrm{~cm}$ de profundidad encontramos que a los $500 \mathrm{~min}$ se tiende al punto de contenido de agua a saturación o se está muy próximo, no siendo asi en los tiempo menores a este.

Tabla 01 Coeficientes de eficiencia y errores de los modelos

\begin{tabular}{cccccccc}
\hline $\begin{array}{c}\text { TIEMPO } \\
(\mathbf{m i n})\end{array}$ & \multicolumn{3}{c}{ MODELOS ENSAYADOS } & \multicolumn{3}{c}{ CÁLCULO DE ERRORES } \\
RELATIVOS & Richards & $\begin{array}{c}\text { Smith- } \\
\text { Parlange }\end{array}$ & $\begin{array}{c}\text { Green } \\
\text { Ampt }\end{array}$ & Experim. & Richards & $\begin{array}{c}\text { Smith- } \\
\text { Parlange }\end{array}$ & $\begin{array}{c}\text { Green } \\
\text { Ampt }\end{array}$ \\
\hline 25 & -8.52 & -6.60 & -11.04 & -8.14 & 4.48 & -23.33 & 26.27 \\
50 & -17.91 & -9.61 & -15.72 & -21.10 & -17.83 & -119.56 & -34.22 \\
100 & -27.45 & -14.21 & -22.45 & -22.00 & 19.84 & -54.82 & 2.00 \\
200 & -37.01 & -21.46 & -32.19 & -28.00 & 24.35 & -30.48 & 13.02 \\
300 & -46.60 & -27.70 & -39.84 & -30.06 & 35.49 & -8.52 & 24.55 \\
500 & -56.22 & -38.97 & -52.28 & -45.12 & 19.74 & -15.78 & 13.70 \\
1000 & -65.93 & -64.91 & -76.17 & -68.11 & -3.31 & -4.93 & 10.58 \\
1500 & -75.89 & -90.08 & -95.42 & -79.09 & -4.22 & 12.20 & 17.11 \\
1600 & -85.88 & -95.00 & -98.94 & -83.02 & 3.33 & 12.61 & 16.09 \\
Cef & 0.98 & 0.94 & 0.99 & 1.00 & & & \\
RMSE & 7.75 & 7.86 & 9.35 & Em & 9.82 & 30.65 & 9.13 \\
\hline
\end{tabular}

Cef $=$ coeficiente de eficiencia (Nash y Sutcliffe, 1970) o coeficiente de correlación con respecto a la línea 1:1; RMSE= Raiz del error cuadrático medio. Em=Error medio 
Como se puede observar el error relativo medio para el modelo Green Ampt, resulta 9.13\%, comparativamente menor que el de Richards que es $9.82 \%$ y SmithParlange en $30.65 \%$ respecto a los resultados experimentales obtenidos por Dahualde G. (2005). Lo mismo ocurre con el coeficiente de eficiencia respecto a la linea 1:1, que son mejores los resultados del modelo Green Ampt 0.99 frente a 0.98 para Richards y 0.94 para Smith-Parlange.

\section{CONCLUSIONES}

De acuerdo a los resultados obtenidos en las simulaciones efectuadas, se puede concluir que el modelo ID basado en la ecuación de Richards de la infiltración que ha sido desarrollado, cumple satisfactoriamente el objetivo de predecir el movimiento del agua en el subsuelo a partir de datos de propiedades físicas de los suelos y condiciones impuestas tipo dirichlet de carga de agua sobre el suelo. Asimismo el modelo es muy versátil, puesto que permite establecer al usuario condiciones como profundidad total de simulación, espaciamiento entre nudos e intervalos de cálculo para la variable temporal.

De las correlaciones y cálculos de errores efectuados se concluye que el modelo de Green Ampt tiene mejores resultados que el modelo de Richards y Smith-Parlange, tomando como referencia los resultados experimentales obtenidos por Dahualde G. (2005).

De los resultados en forma representativa a todos los modelos y resultados experimentales citados se observa que en los instantes iniciales el frente mojado avanza rápidamente en la dirección vertical, lo que se refleja en la pendiente que inicialmente presenta la curva, debido al acelerado aumento del contenido volumétrico de agua con respecto a la profundidad. Luego el frente mojado avanza cada vez más lento, lo que se manifiesta en una pendiente menos pronunciada.

Es muy importante el hecho que se requiere simular el proceso de infiltración de agua en el suelo en diferentes escenarios por ejemplo la agricultura, conservación de suelo, etc. Asimismo este modelo de Richards (1931) nos puede determinar la velocidad de infiltración la misma que influye en el escurrimiento superficial y a su vez en el proceso de erosión hidrica. Otra importante utilidad radica en los trabajos de manejo de recursos hidricos en obras urbanas referidas a diseño de alcantarillas, zanjas de infiltración, etc.

Se propone que los resultados obtenidos con este modelo sean validados con datos experimentales de campo o laboratorio en las diferentes regiones del pais.

\section{AGRADECIMIENTOS}

Agradecimiento especial al programa de post-grado en la Especialidad de Recursos Hidricos de la Universidad Nacional Agraria La Molina Lima y la Escuela de Ingeniería Geológica-Geotecnia de la Universidad Nacional Jorge Basadre Grohmann de Tacna.

\section{REFERENCIAS BIBLIOGRÁFICAS}

Chapra, S. C.; Canale, R. P. Métodos Numéricos para Ingenieros. Mc. GRAW-HILL INTERAMERICANA EDITORES S.A. de C.V.p. 851-957, Marzo 2003.

Clemmens, A. J., Discusion of dimensionaless solutions of border irrigation advance by STREALKOFF, T.

Katapodes, N., (1977). In Journal of irrigation and Drainage Engineering. ASCE, vol. 104, num. 3, p. $337-$ 339,1978 .

Ortiz, J.: Nikolskii, I.; Palacios, O.; Acosta, R.; Pérdidas de Agua de Riego por Precolación Profunda Durante el Proceso de Infiltración. Revista TERRA Latinoamericana, Revista Mexicana de la Ciencia del Suelo, vol 17, num 2, p. 115-124, junio 1999.

Reyna, M., Reyna, T.; Interacción de los procesos de escorrentia e infïltración.

ttp://www.unesco.org.uy/phi/libros/congreso/36reyna Il Congreso Argentino de Hidrogeología, IV Seminario Hispano Argentino sobre temas actuales de la Hidrología Subterranea. Santa Fe, Argentina 28 de Setiembre al 1 de Octubre de 1999.

Saucedo, H., Fuentes, C., Zavala, M.; El sistema de ecuaciones de Saint-Venat y Richards del riego por gravedad: 2. Acoplamiento numérico para la fase de avance en el riego por melgas. Revista Ingeniería Hidráulica en México, vol XX, num 2, p. 109-119, Abril-Junio 2005.

\section{Correspondencia:}

Edwin Pino Vargas

Ciudad Universitaria - Fundo "Los Granados"

Av. Miraflores $\mathrm{s} / \mathrm{n}$. Tacna. Perú

epino68@hotmail.com

Jesús Abel Mejía Marcacuzco

Universidad Nacional Agraria La Molina

Lima. Perú

jabel@lamolina.edu.pe 